\title{
Recognition without picture identification: Geons as components of the pictorial memory trace
}

\author{
ANNE M. CLEARY, MOSES M. LANGLEY, and KEVIN R. SEILER \\ Iowa State University, Ames, Iowa
}

\begin{abstract}
Participants viewed a list of black-and-white line drawings and were then presented with a picture fragment identification task in which half of the fragments corresponded to studied pictures and half corresponded to unstudied pictures. In addition to trying to identify each picture fragment, participants gave a rating to indicate the likelihood that the fragment came from a studied picture. When participants could not identify the picture fragments, they were still able to discriminate between fragments that came from studied pictures and fragments that came from unstudied pictures (as shown by their recognition ratings), but only when the fragments contained information about the geometric components (geons) that underlay the original pictures. No recognition without identification was found when the fragments contained only line segment information.
\end{abstract}

A longstanding premise among memory researchers is that the memory trace for an item's presentation on a study list is composed of the constituent features that form the item (e.g., Tulving \& Bower, 1974). This idea is central to many mathematical models of memory, including models of recognition memory (e.g., Clark \& Gronlund, 1996). However, little is known about the types of features that may actually be present in human memory traces. In the present study, we explore the nature of some of the features that may be present in memory traces for studied pictures.

A paradigm that has proven useful for probing the features of the memory trace is that which has been used to study the phenomenon of recognition without identification. In studies of recognition without identification (Cleary \& Greene, 2000; Peynircioğlu, 1990), participants study a list of words (e.g., RAINDROP, AMETHYST) and are then presented with a test list containing word fragments. Half of these word fragments correspond to studied words (e.g., R_I__R_P), and half correspond to unstudied words (e.g., S_Q_E__E). For each fragment presented at test, participants are first asked to try to complete the fragment. Regardless of whether they can do so, they are then asked to rate the likelihood that the fragment comes from a studied word. When only the ratings given to fragments that cannot be identified are examined, a significantly higher mean rating is found for fragments corresponding to studied words than for those corresponding to unstudied words. This finding has been

The authors thank Eric E. Cooper for the line drawings used in the present study. We also thank Amanda Albertson, Jessica Brower, Josh Carlson, Glenn Casner, Jeneice Dickey, Amy Hatten, Lisa Lancaster, Erin MacCoy, Katie Mulford, and Anthony Phillips for their assistance with data collection. Correspondence concerning this article should be addressed to A. M. Cleary, Department of Psychology, Iowa State University, W. 112 Lagomarcino, Ames, IA 50011 (e-mail: acleary@iastate.edu). shown to be a small but reliable effect, and it persists even when surface features, such as presentation modality and letter case, have been changed from study to test (e.g., Cleary \& Greene, 2000).

The recognition without identification paradigm is useful for probing the features of memory traces because it presents a means of isolating particular features of recognition test items and then examining recognition that is based solely on the availability of those features. Note that, in the aforementioned studies of recognition without identification, only particular letters in particular locations (e.g., R__DR__P) were presented on the recognition test, yet people could still recognize unidentifiable fragments as belonging to studied words, even when the surface features had changed from study to test. This suggests that people relied on memory for the letter features of studied words when recognizing word fragments in the absence of their identification. Thus, a word's letter information may be a type of feature stored in the memory trace for the word's occurrence on a study list.

If the recognition without identification paradigm is applied to other stimulus realms, it may prove useful for identifying other types of features that are present in memory traces for study list items. In fact, Cleary (2004) used the recognition without identification paradigm to examine whether recognition could occur solely on the basis of a word's orthographic, phonological, or semantic features. After studying a list of words, participants were presented with a cued recall task in which half of the test cues resembled studied words and half resembled unstudied words on one particular dimension. For each cue presented at test, participants first attempted to use the cue to recall a study list item resembling it. Then, regardless of whether they could recall a study list item, participants rated the test cue using a scale of 0 (definitely not similar to a studied word) to 10 (definitely similar to a studied word). When ratings given to the test 
cues that could not be used to recall studied words were examined, mean ratings were significantly higher for cues resembling studied words than for cues resembling unstudied words. This recognition without recall effect occurred when the test cues were orthographic in nature, such as when cheetohs served as a cue for recalling cheetah (Cleary, 2004, Experiments 1 and 2), when they were phonological in nature, such as when laughed served as a cue for recalling raft (Experiment 3), and when they were semantic in nature, such as when jaguar served as a cue for recalling cheetah (Experiment 4). These findings suggest that some word features that may be present in memory traces for study list words are the orthographic, phonological, and semantic characteristics of those words.

In the present study, we used the recognition without identification paradigm to examine what types of features may be found in memory traces for pictorial information. For ideas about how pictures might be segmented into sets of isolable features in memory, one can look to theories of object identification. Some theorists of object identification argue that the basic features of visual objects are their geometric component parts (e.g., Biederman, 1987). According to this approach, when people see an object, they first detect its component parts and then determine its identity on the basis of how the parts interrelate. Biederman argued that the basic components of any object are simple, three-dimensional shapes he called geons. Cones, pyramids, cylinders, and brick-like structures are some examples. If this is indeed how pictorial information is processed by the visual object identification system, one might expect some features of the memory trace for a studied picture to be the geons that appeared in that picture.

However, not everyone agrees that visual object identification results from the segmentation of objects into components. According to view-based theories (see Hummel, 2000 , for a review), people come to identify everyday objects by matching the incoming visual images to wholeimage representations stored in memory. Thus, many view-based theorists consider visual object representations to be holistic in nature, rather than componential. It is worth noting that the two approaches are not mutually exclusive; in fact, some evidence suggests that both holistic and componential approaches can account for different types of human visual identification (Cooper \& Wojan, 2000).

Perhaps the most compelling support for componential object representations has stemmed from the following logic: It should be easier for the visual system to detect the places where an object can be broken down into component parts when the junction points (where component parts join one another) are included in a picture fragment than when the junction points are deleted. Indeed, people have an easier time identifying pictures from fragments whose junction points have been left intact than from fragments whose junction points have been deleted (Biederman, 1987).

The present study served both as an attempt to extend the recognition without identification phenomenon to the realm of picture recognition and as an exploration of the nature of pictorial memory traces. For the latter goal, we explicitly manipulated the presence or absence of junction points in the picture fragments that we presented at test. The specific purpose of this was to examine whether the componential representations thought to give rise to the junction point advantage shown by Biederman (1987) might also give rise to recognition without picture identification. After participants studied a list of black-and-white line drawings, they were presented with a test list containing picture fragments corresponding to studied and unstudied pictures. Half of the participants received picture fragments containing junction points, and half received picture fragments containing the same number of pixels but no junction points (see Figure 1 for examples). For each picture fragment presented at test, participants first attempted to identify the picture and then rated the likelihood that the fragment came from a studied picture. The interest in the present study was in ratings given to unidentified picture fragments.

In comparing ratings given to picture fragments with and without junction points, we used the following logic. If an unidentifiable picture fragment can activate a memory trace for the structural aspects of a recently presented picture, and if that trace is componential in nature (i.e., composed of geometric components), one might expect to find recognition without picture identification only when the fragments contain junction points (which would allow at least some segmentation of each fragment into components), and not when the junction points have been deleted. However, if the memory trace for the structural aspects of a recently presented picture is holistic in nature, then whether or not junction points are present in the picture fragments should not matter. That is, one might expect to find recognition without picture identification both when junction points are present and when they are absent in the picture fragments used at test.

The idea that an unidentifiable picture fragment might activate an existing structural representation in memory is not without precedent. There is reason to suspect that structural representations that are pictorial in nature can become activated in the absence of their conscious identification. Bar and Biederman (1998) found that flashing masked pictures too quickly for identification to take place still led to priming on a later picture identification task. Because such priming was absent when the targets were objects with different shapes but the same names as those of the primes, this form of priming appears to have resulted from the activation of visual shape representations. It is plausible that the activation of such structural representations might lead to an ability to recognize that an unidentifiable picture fragment belongs to a recently presented picture.

Moreover, there is reason to suspect that the recognition without identification phenomenon can be extended to the realm of picture recognition. In some of their experiments examining the relationship between perceptual fluency and episodic picture recognition, Snodgrass, 


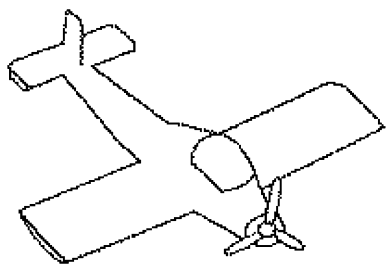

$1 \mathbf{A}$

2A
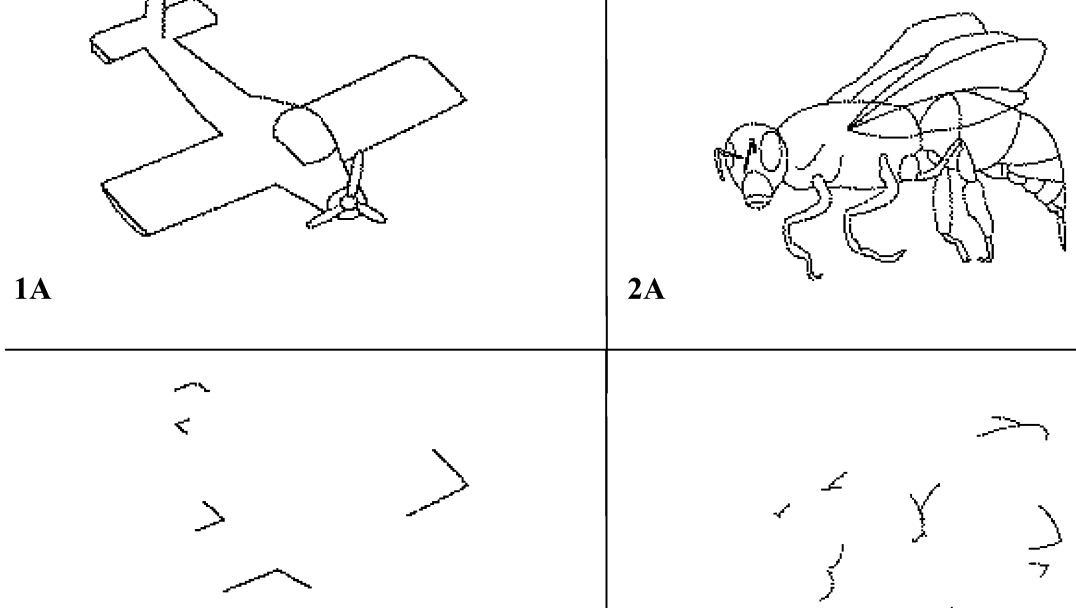

1B

2B
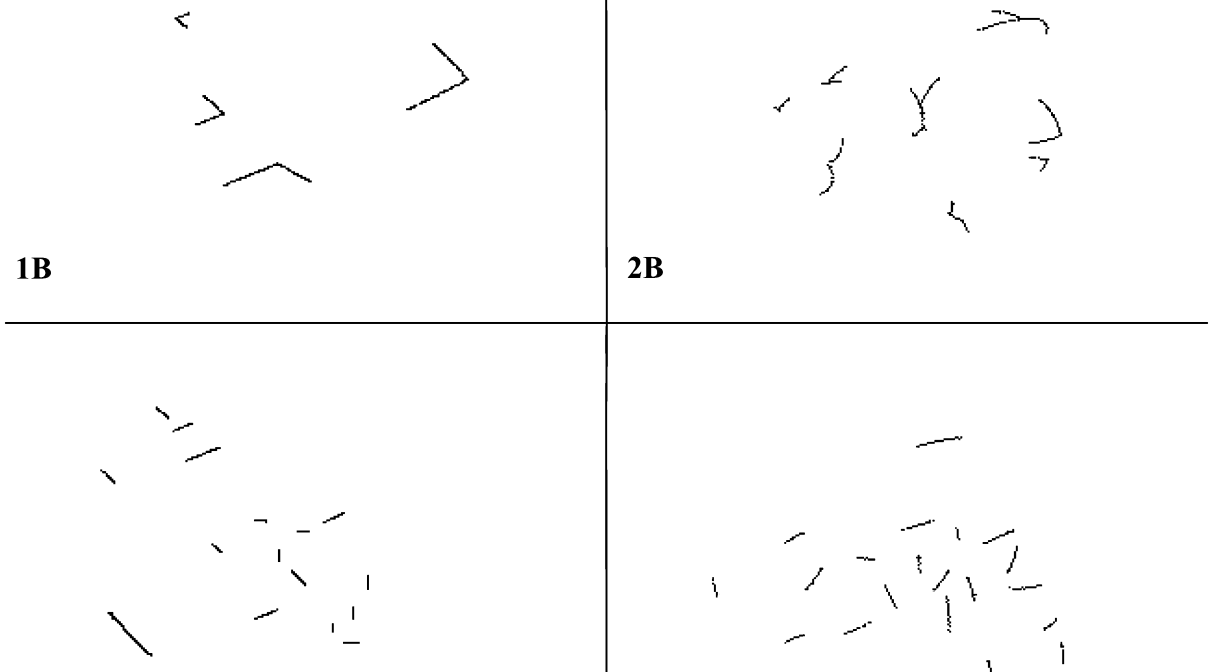

$1 \mathrm{C}$

$2 \mathrm{C}$

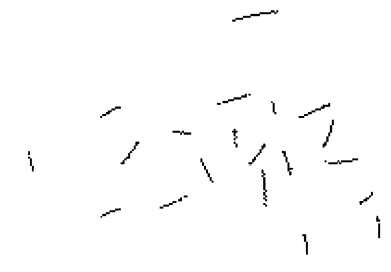

Figure 1. Sample stimuli from the present study. The items shown in $1 \mathrm{~A}$ and $2 \mathrm{~A}$ are pictures that might have been used in a given study list ("airplane" and "bee"). 1B and 2B show the geon fragments corresponding to the pictures shown in $1 \mathrm{~A}$ and $2 \mathrm{~A}$, respectively. $1 \mathrm{C}$ and $2 \mathrm{C}$ show the non-geon fragments. Fragments were always $10 \%$ of their original pictures in total pixel number. Thus, geon and non-geon fragments corresponding to the same picture were equated according to their total number of pixels.

Hirshman, and Fan (1996) reported the $d^{\prime}$ values obtained from yes-no recognition judgments given to picture fragments that could and could not be identified at test. The $d^{\prime}$ scores for unidentified picture fragments tended to be so low that they did not differ significantly from zero; however, although it was not the focus of their study, Snodgrass et al. (Experiment 3) reported at least one condition in which the $d^{\prime}$ score obtained for unidentified picture fragments was significantly greater than zero. But it should be noted that unlike in the present study, Snodgrass et al. did not explicitly manipulate the presence or absence of junction points in the picture fragments that were used. Thus, although there is some reason to suspect that the recognition without identification phenomenon can be extended to the realm of picture recognition, it is unclear what role, if any, the presence or absence of junction points would play in obtaining the effect.

\section{METHOD}

\section{Participants}

One hundred forty-five Iowa State University undergraduates participated in exchange for extra credit in an introductory psychology course.

\section{Materials}

Stimuli were 48 black-and-white line drawings similar to those used by Biederman (1987). The drawings depicted common animate and inanimate objects, such as an airplane, a bee, a hammer, or a piano. Fragments corresponding to each of these pictures were constructed by deleting portions of the original pictures. Each fragment contained $10 \%$ of the pixels present in the original picture (the specific percentage was chosen to ensure that the fragments would be difficult to identify). The fragments were explicitly created to resemble Biederman's (1987) "recoverable" and "nonrecoverable" objects. In the "recoverable" (geon) fragments, some of the junction points (places where line segments intersect) were left intact. In the "nonrecoverable" (non-geon) fragments, only midsegments of lines 
were apparent; no junction points were left intact (see Figure 1 for examples).

Within a given fragment condition, all of the 48 fragments were used as test stimuli, and the pictures corresponding to 24 of these fragments were randomly chosen by a computer program to be used as study-list stimuli for each participant. All stimuli were sized to fit within a space of $250 \times 250$ pixels.

\section{Procedure}

All segments of the experiment were conducted on a computer, and participants were randomly assigned to the geon and non-geon conditions. As has been done in prior studies of the recognition without identification phenomenon (e.g., Cleary \& Greene, 2000), several study-test segments were used, and the general procedure was explained to participants before beginning. Importantly, participants were given identical instructions in both fragment conditions. They were told that they would be viewing a list of black-andwhite line drawings on the computer, and that their memory for the drawings would be tested. They were also told that, on the memory test, they would be presented with fragments of pictures to identify, and that some of the fragments would correspond to pictures that were studied and some would correspond to pictures that were not studied. They were told that, in addition to attempting to identify each of the picture fragments, they would be asked to decide whether they thought the fragment corresponded to a picture that was seen at study, but that more specific instructions would be given to them after the presentation of the first study list. They were told nothing specific about the nature of the test fragments.

The participants completed three study-test segments, each containing a study list of eight pictures followed by a test list of 16 picture fragments. For each participant, the computer program randomly assigned study and test stimuli to blocks (first, second, or third block), and the order of presentation within blocks was randomly determined. In a given study segment, each picture appeared in the top left corner of the computer monitor for a duration of $2 \mathrm{sec}$ followed by a $1-\mathrm{sec}$ interstimulus interval. At test, each picture fragment appeared in the top left corner of the computer monitor and remained on the screen as the questions pertaining to it were presented. Upon being presented with a fragment, the participants were first prompted to try to identify the picture from which the fragment was created. They were asked, "Can you identify this picture? If so, type its name." The participants could respond either by typing in a word and pressing the return key, or by simply pressing the return key. After doing so, they were prompted to rate the picture fragment, indicating how likely they thought it was to have come from a picture that was seen in the study list. They were instructed to use a scale of 0 (definitely not from the study list) to 10 (definitely from the study list). The participants could respond only by typing an integer between 0 and 10 and then pressing the return key. In addition, they were explicitly instructed to rate every fragment, even those that could not be identified.

If a participant had correctly identified a picture in response to the first question (by typing the correct answer), then, after giving a rating, he or she was immediately presented with the next test fragment. If, however, the participant had not typed in the correct name, then, after giving a rating, he or she was given a second chance to do so. Here, participants were instructed that if they could not identify the picture, they should take a guess. Whenever this second chance led to a correct identification, the rating given was classified as belonging to an identified item. This was done to ensure that any findings would not be attributable to participants withholding responses during the initial identification portion of each test trial. The participants were told not to guess on the first attempt at identification; this was to ensure that when rating a particular fragment whose identity was unclear, the participants rated the likelihood that the fragment came from the study list, rather than the likelihood that a particular item brought to mind as a guess appeared in the list.
Prior to analysis, the data were checked for spelling errors and for the use of alternative names to identify pictures (e.g., typing pistol instead of gun). Any response that accurately identified a picture fragment but was either spelled incorrectly or was an alternative name for the object in question was classified as an instance of correct identification.

\section{RESULTS}

In all of the analyses presented here, a .05 significance criterion was used. The data for all experimental conditions are reported in Tables 1, 2, and 3. Table 1 lists the proportions of picture fragments correctly identified in each condition. A $2 \times 2$ study status (studied vs. unstudied) $\times$ fragment type (geon vs. non-geon) mixed analysis of variance (ANOVA) revealed a significant main effect of study status, such that participants identified more fragments of studied pictures than of unstudied pictures $\left[F(1,143)=491.16, M S_{\mathrm{e}}=0.02, p<.001\right]$. There was also a significant main effect of fragment type, such that more geon fragments were identified than non-geon fragments $\left[F(1,143)=109.62, M S_{\mathrm{e}}=0.02\right.$, $p<.001]$. Note that this is consistent with Biederman's (1987) theory of object identification. A significant interaction between study status and fragment type was also found $\left[F(1,143)=16.62, M S_{\mathrm{e}}=0.02, p<.001\right]$. This interaction was such that the tendency to identify more studied than unstudied items was greater when geon fragments were used than when non-geon fragments were used.

We turn now to the data of interest - the recognition ratings given to the picture fragments that could not be identified (Table 2). A $2 \times 2$ study status (studied vs. unstudied) $\times$ fragment type (geon vs. non-geon) mixed ANOVA performed on ratings given to unidentified picture fragments revealed a significant interaction $[F(1,143)=4.80$, $\left.M S_{\mathrm{e}}=0.49, p<.05\right]$. This interaction was such that the difference between ratings given to fragments of studied pictures and ratings given to fragments of unstudied pictures was larger when geon fragments were used than when non-geon fragments were used. As can be seen in Table 2, when geon fragments were used, participants gave higher ratings to unidentifiable fragments of studied pictures than to those of unstudied pictures $[t(72)=$ $2.86, p<.01]$. However, when non-geon fragments were used, no such discrimination was shown between unidentifiable fragments of studied pictures and unidentifiable fragments of unstudied pictures $[t(71)=-0.42$, n.s.]. Thus, a recognition without picture identification effect was found, but only when the fragments contained junc-

Table 1

Mean Proportion Correctly Identified as a Function of Study Status and Fragment Type

\begin{tabular}{ccccccc}
\hline \multirow{2}{*}{ Condition } & \multicolumn{2}{c}{ Studied } & & \multicolumn{2}{c}{ Unstudied } \\
\cline { 2 - 3 } & $M$ & & $S D$ & & $M$ & $S D$ \\
\hline Geon fragments & .58 & .19 & & .13 & .08 \\
Non-geon fragments & .35 & .18 & & .04 & .05 \\
\hline
\end{tabular}


Table 2

Mean Recognition Ratings as a Function of Identification, Study Status, and Fragment Type

\begin{tabular}{|c|c|c|c|c|c|c|c|c|}
\hline \multirow[b]{3}{*}{ Condition } & \multicolumn{4}{|c|}{ Identified } & \multicolumn{4}{|c|}{ Unidentified } \\
\hline & \multicolumn{2}{|c|}{ Studied } & \multicolumn{2}{|c|}{ Unstudied } & \multicolumn{2}{|c|}{ Studied } & \multicolumn{2}{|c|}{ Unstudied } \\
\hline & $M$ & $S D$ & $M$ & $S D$ & $M$ & $S D$ & $M$ & $S D$ \\
\hline Geon fragments & 7.97 & 1.90 & 3.26 & 2.15 & 3.96 & 1.29 & 3.65 & 1.25 \\
\hline Non-geon fragments & 8.09 & 1.71 & 4.10 & 3.35 & 4.22 & 1.48 & 4.27 & 1.39 \\
\hline
\end{tabular}

tion points. Because multiple study-test segments were used in the present study, these data were analyzed as a function of segment (segments 1, 2, and 3), and it was found that recognition performance did not change as a function of segment in either fragment condition. ${ }^{1}$ Furthermore, because the levels of identification differed between the geon and non-geon conditions, the data were examined as a function of identification accuracy. As can be seen in Table 3, the pattern revealed in the overall analysis was present at each level of identification accuracy. Thus, the pattern does not appear to be the result of item selection effects. In addition, we computed the correlations between recognition without identification performance and identification rates. No significant correlations were found. ${ }^{2}$

Also coming out of the $2 \times 2$ ANOVA on ratings given to unidentified picture fragments was a main effect of fragment type $\left[F(1,143)=4.53, M S_{\mathrm{e}}=3.20, p<.05\right]$. This main effect was such that ratings given to non-geon fragments were higher overall than ratings given to geon fragments. One explanation for this may be that, because the geon fragments were, on average, easier to identify, participants were more likely to use a fluency heuristic (e.g., Whittlesea, Jacoby, \& Girard, 1990) to attribute the fact that a geon fragment could not be identified to its having not been studied. The main effect of study status was not significant $\left[F(1,143)=2.42, M S_{\mathrm{e}}=0.49, p=\right.$ $.12]$, presumably because of the contribution of performance in the non-geon condition.

Table 3

Mean Recognition Ratings as a Function of Identification Performance

\begin{tabular}{crcccccc}
\hline \multirow{2}{*}{$\begin{array}{c}\text { S Studied Items } \\
\text { Identified }\end{array}$} & \multicolumn{3}{c}{ Geon Fragments } & & \multicolumn{3}{c}{ Non-Geon Fragments } \\
\cline { 2 - 4 } \cline { 6 - 8 } & $N$ & Studied & Unstudied & & $N$ & Studied & Unstudied \\
\hline Under $20 \%$ & 5 & 3.74 & 3.24 & & 15 & 3.99 & 4.39 \\
$20 \%-50 \%$ & 13 & 4.27 & 3.78 & & 40 & 4.15 & 4.11 \\
$50 \%-75 \%$ & 44 & 4.08 & 3.81 & & 17 & 4.60 & 4.57 \\
$76 \%-88 \%$ & 11 & 3.20 & 3.06 & & 0 & - & - \\
\hline
\end{tabular}

Note-In order to compare recognition ratings at similar levels of identification performance, the data were segmented according to where large numbers of participants in both the geon and non-geon conditions fell in terms of identification performance. Note that in both the geon and non-geon conditions, 57 participants identified between $20 \%$ and $75 \%$ of the studied fragments. However, whereas in the geon condition the majority of these participants identified over $50 \%$, in the non-geon condition, the majority of the participants identified under $50 \%$. No participants in the non-geon condition identified over $75 \%$, and no participants in the geon condition identified over $88 \%$.
Following Cleary and Greene (2000), a $2 \times 2$ identification status (identified vs. unidentified) $\times$ study status (studied vs. unstudied) repeated measures ANOVA was performed on ratings given in the geon condition (where recognition without identification was found) to compare recognition with and without identification. The analysis replicated the pattern reported by Cleary and Greene (2000). First, there was a significant main effect of identification status $\left[F(1,65)=84.92, M S_{\mathrm{e}}=\right.$ $2.37, p<.001]$, such that ratings were higher for identified than for unidentified items; as is evident in Table 2, this appears to be due to the extremely high ratings given in the studied, identified category. There was also a main effect of study status $\left[F(1,65)=170.70, M S_{\mathrm{e}}=2.41\right.$, $p<.001]$; ratings were higher overall for studied than for unstudied items. The interaction between identification status and study status was significant as well $[F(1,65)=$ $\left.136.83, M S_{\mathrm{e}}=2.41, p<.001\right]$. Not surprisingly, discrimination between studied and unstudied picture fragments was better when those fragments could be identified than when they could not be identified.

\section{DISCUSSION}

In the present study, participants were able to discriminate between unidentifiable fragments of studied pictures and unidentifiable fragments of unstudied pictures, but only when the fragments contained junction points. When no junction points were present in the picture fragments used at test, participants showed no such discrimination. Not only do the present findings extend the generality of the recognition without identification effect first reported by Peynircioğlu (1990), but they also help to establish some of its boundaries.

The fact that recognition without picture identification occurred only when the picture fragments contained junction points suggests that it resulted from the activation of pictorial memory traces that were componential in nature. Presumably, junction points enable the segmentation of an image into component parts, whereas the absence of junction points makes such segmentation difficult (Biederman, 1987). If the pictorial memory traces for the list items used in the present study were holistic in nature, the presence or absence of junction points would not be expected to impact whether a recognition without identification effect was found.

That pictorial memory traces may be componential in nature is consistent with the long-held assumption among memory researchers that memory traces consist of sets of features (e.g., Tulving \& Bower, 1974). In this regard, the present findings are important because they give clues about the kinds of features that may make up memory traces. Biederman (1987) suggested that a junction point advantage in the identification of picture fragments indicates that objects are segmented into their constituent geometric components (geons) prior to identification. The present finding that recognition without picture identification emerged only when the test frag- 
ments contained junction points may indicate that memory representations containing geons were involved. If so, the geon may represent a type of feature that can be present in a memory trace for a recently experienced event.

Some may be concerned that the critical difference between the fragments containing junction points and the fragments not containing junction points is not the presence or absence of geon information, but rather the fact that fragments containing junction points are simply more distinctive (more discriminable from one another) than fragments not containing junction points (see Figure 1). It is possible that the recognition without identification shown in the junction point condition is related to the distinctiveness of the fragments; it should be easier to distinguish between studied and unstudied items when the items themselves are more distinguishable from one another. However, this question still remains: What makes the items in the junction point condition more distinguishable from one another, and on what basis are participants discriminating between unidentified studied and unidentified unstudied items in this condition?

It is very likely that what makes these items distinct is the fact that at least some of the component parts of the pictures are discernable, and that the human visual identification system is inclined toward segmenting images into component parts. If the human visual identification system were not inclined toward segmenting images into component parts, one might expect a fragment containing junction points to be no more distinctive than a fragment containing an equivalent number of pixels but no junction points. Furthermore, what probably allows participants to discriminate between unidentified studied and unidentified unstudied fragments in the junction point condition is the availability of component shape information: Participants recognize some component shapes as having appeared (or having not appeared) in the study list.

In short, the present findings are consistent with the claim that pictorial representations are componential in nature. There is something about picture fragments that can be easily segmented into components that allows memory for their recent occurrence to take place in the absence of their identification.

\section{REFERENCES}

Bar, M., \& Biederman, I. (1998). Subliminal visual priming. Psychological Science, 9, 464-469.

BIEDERMAN, I. (1987). Recognition-by-components: A theory of human image understanding. Psychological Review, 94, 115-147.

Clark, S. E., \& Gronlund, S. D. (1996). Global matching models of recognition memory: How the models match the data. Psychonomic Bulletin \& Review, 3, 37-60.
Cleary, A. M. (2004). Orthography, phonology, and meaning: Word features that give rise to feelings of familiarity in recognition. Psychonomic Bulletin \& Review, 11, 446-451.

CleARY, A. M., \& GREene, R. L. (2000). Recognition without identification. Journal of Experimental Psychology: Learning, Memory, \& Cognition, 26, 1063-1069.

CoOPER, E. E., \& WoJAN, T. J. (2000). Differences in the coding of spatial relations in face identification and basic-level object recognition. Journal of Experimental Psychology: Learning, Memory, \& Cognition, 26, 470-488.

HUMMEL, J. E. (2000). Where view-based theories break down: The role of structure in shape perception and object recognition. In E. Dietrich \& A. B. Markman (Eds.), Cognitive dynamics: Conceptual and representational change in humans and machines (pp. 157-185). Mahwah, NJ: Erlbaum.

PeYNIRCIOǦLU, Z. F. (1990). A feeling-of-recognition without identification. Journal of Memory \& Language, 29, 493-500.

Snodgrass, J. G., Hirshman, E., \& Fan, J. (1996). The sensory match effect in recognition memory: Perceptual fluency or episodic trace? Memory \& Cognition, 24, 367-383.

TULVING, E., \& BOWER, G. H. (1974). The logic of memory representations. In K. W. Spence \& J. T. Spence (Eds.), The psychology of learning and motivation (Vol. 7, pp. 265-300). New York: Academic Press.

Whittlesea, B. W. A., Jacoby, L. L., \& Girard, K. A. (1990). Illusions of immediate memory: Evidence of an attributional basis for feelings of familiarity and perceptual quality. Journal of Memory \& Language, 29, 716-732.

\section{NOTES}

1. Reviewers of a previous version of this article were concerned that participants might study differently if made aware of the nature of the test fragments. However, recognition performance did not change as a function of practice. For ratings given to unidentified fragments in the geon condition, a $2 \times 3$ study status (studied vs. unstudied) $\times$ list (first vs. second vs. third) repeated measures ANOVA revealed no significant interaction $[F(1,60)=0.91$, n.s.] and no significant main effect of list $[F(1,60)=0.38$, n.s. $]$. The only main effect shown was for study status $\left[F(1,60)=9.55, M S_{\mathrm{e}}=1.51\right]$; ratings were higher overall for fragments of studied pictures than for fragments of unstudied pictures. The same analysis performed on ratings given in the non-geon condition revealed no significant interaction $[F(1,71)=0.00$, n.s.], no main effect of list $[F(1,71)=0.68$, n.s. $]$, and no main effect of study status $[F(1,71)=$ 0.18, n.s.].

2. The difference between the mean rating for unidentified studied items and the mean rating for unidentified unstudied items was computed for each participant; then the correlation between these differences (which can be seen as indexes of recognition without identification) and the mean proportions of identified, studied items (which can be seen as indexes of identification performance) were computed for the geon condition $[r(72)=-.11$, n.s. $]$ and for the non-geon condition $[r(71)=-.18, \mathrm{n} . \mathrm{s}$.$] . The correlation between the ratings differences$ (again, indexes of recognition without identification) and the mean proportions of identified, unstudied items (which can be seen as another means of indexing identification performance) was also computed for both the geon condition $[r(72)=-.06$, n.s.], and the non-geon condition $[r(71)=.02$, n.s. $]$.

(Manuscript received February 26, 2002; revision accepted for publication September 10, 2003.) 